\title{
ENSO dynamics: Low-dimensional-chaotic or stochastic?
}

\author{
T. Živković ${ }^{1}$ and K. Rypdal ${ }^{2}$ \\ Received 16 October 2012; revised 3 January 2013; accepted 10 January 2013; published 12 March 2013.
}

[1] We apply a test for deterministic, low-dimensional, and nonlinear dynamics to the Niño 3 time series for the El Niño Southern Oscillation (ENSO). The test is positive if the time series includes the seasonal variation, but negative if it only represents the anomaly, i.e., the deviation from the seasonal cycle. The results indicate that deterministic, low-dimensional, and nonlinear dynamics in ENSO is associated with the seasonal cycle, and that the dynamics determining the interannual timing and strength of El Niño/La Niña episodes is high-dimensional/stochastic. Application of stochastic forcing to a time-delay equation for equatorial-wave dynamics can reproduce stochastic dynamics and other important aspects of ENSO. Without such stochastic forcing, this model yields deterministic, low-dimensional dynamics. With stochastic forcing, our test still yields such dynamics if the seasonal cycle is retained, but does not if the annual cycle is subtracted before the test is applied. The model results illustrate that the seasonal variability can be governed by low-dimensional nonlinear dynamics, while the interannual variability associated with ENSO is dominated by the stochastic forcing.

Citation: Živković, T., and K. Rypdal (2013), ENSO dynamics: Low-dimensional-chaotic or stochastic?, J. Geophys. Res. Atmos., 118, 2161-2168, doi:10.1002/jgrd.50190.

\section{Introduction}

[2] Prediction is the ultimate goal of meteorology and climatology, and the issue of predictability is crucial. Prediction in these disciplines is mostly probabilistic, but there may be different rationales for a probabilistic description. These rationales are intimately linked to the various meanings of the concepts of determinism, deterministic chaos, and stochasticity. The fundamental laws of classical physics are deterministic, since the future and past of the state of such a system is uniquely given by the state at a given time. The majority of models of weather and climate are deterministic in this sense. Predictability in chaotic systems is limited by the largest positive Lyapunov exponent. This may require a probabilistic description, not only in systems with a large number of degrees of freedom, but also in simple, lowdimensional, nonlinear systems. When dealing with data in climatology, either from observation or from large-scale simulations, the climate dynamicist will have to ask the question of whether prediction for the system/phenomenon of interest is better served by a low-dimensional chaotic model of climate variability, or by a high-dimensional (stochastic) model. Hence, there is a demand for methods by which it is possible to decide from the observation data whether the system dynamics can be uniquely projected onto an attractor in a low-dimensional phase space. If the system is autonomous and the attractor of the trajectory has

\footnotetext{
${ }^{1}$ Swedish Institute of Space Physics, University of Uppsala, Sweden.

${ }^{2}$ Department of Mathematics and Statistics, University of Troms $ø$, Norway.

Corresponding author: T. Živković, Swedish Institute of Space Physics, Box 537, Uppsala, 751 21, Sweden. (tatjana.zivkovic@irfu.se)

(C)2013. American Geophysical Union. All Rights Reserved.

2169-897X/13/10.1002/jgrd.50190
}

dimension $d$, the Takens' time-delay method [Takens, 1981] can be used to construct an $m>2 d$-dimensional embedding space on which the attractor can be mapped continuously and one to one. In practice this method works only if the attractor dimension $d$ is reasonably low. Dynamic systems with a large number of independent or weakly dependent degrees of freedom can only be described either by large-scale numerical simulation or by stochastic methods. For such systems, the phase-space attractor is also high dimensional and cannot be mapped one to one onto a lowdimensional time-delay embedding space. The computation of attractor dimension then typically fails to converge when embedding dimension $m$ is increased, but such convergence can be difficult to detect if the time series is short. Kaplan and Glass [1992, 1993] devised a direct test for the existence of low-dimensional deterministic dynamics which is useful for short time series. This is the kind of test that will be employed in this paper to the instrumental time series for the El Niño Southern Oscillation (ENSO).

[3] The problem of determinism versus stochasticity in ENSO has been a subject of research for more than two decades, and conflicting results have been reported. Bauer and Brown [1992] analyzed Eastern tropical Pacific sea surface temperature (SST) anomalies via the reconstruction of the attractor by means of singular spectrum analysis and found indications of low-dimensional dynamics. Elsner and Tsonis [1993] found that the Southern Oscillation index (SOI) exhibits signatures of nonlinear dynamics by employing a nonlinear prediction algorithm to the SOI time series and to surrogate data exhibiting the same correlation structure but devoid of the nonlinearities. On the other hand, Schreiber and Schmitz [2000], using a more accurate scheme, came to the conclusion that the linear null hypothesis cannot be rejected on the basis of the SOI time series. The 
same conclusion was drawn by Binder and Wilches [2002] from the application of tests to the SOI which are similar to those we employ in the present paper. The data analyzed in all these papers are anomalies, i.e., the seasonal cycle is eliminated by considering the departure from the mean value for the season. There is a general consensus that ENSO is strongly and nonlinearly coupled and phase synchronized with the annual cycle [Stein et al., 2011], so this is not an issue of controversy. The response of the tropical Pacific to the seasonal forcing is nonlinear, and the seasonal timing of ENSO episodes (its phase) is nonlinearly connected to the seasonal forcing. But that does not imply that the trigging of a Niño episode one year, and not another year, is a result of low-dimensional, nonlinear dynamics alone. In this paper, we explore the significance of this distinction by analyzing the Niño 3 index, which is the average of the SST in the region $150^{\circ} \mathrm{W}-90^{\circ} \mathrm{W}$ and $5^{\circ} \mathrm{N}-5^{\circ} \mathrm{S}$, both with the seasonal cycle included and as an anomaly with the cycle subtracted. For the former, we find low-dimensional determinism and nonlinearity, and for the latter, we do not.

[4] The remainder of the paper is structured as follows. In section 2 we briefly describe a time-delay equation for equatorial wave dynamics and ENSO, and in section 3 we review the tests for low-dimensional determinism and nonlinearity. In section 4 these techniques are first applied to the SOI and fail to reveal low-dimensional deterministic dynamics, in agreement with Binder and Wilches [2002]. We then employ them to the Niño 3 data and to numerical solutions of the time-delay equation with and without seasonality subtracted and with and without stochastic forcing. We also apply a superposed-epoch analysis to these data to highlight the characteristic waveforms of ENSO episodes as manifested in the Niño 3 signal. The implications of our findings are discussed in this section and summarized in section 5 .

\section{A Time-Delay Equation for ENSO}

[5] A large body of literature is concerned with the dynamical modeling of ENSO (a review can be found in Dijkstra [2010]). An interesting class of models is described as delaydifferential equations, which are linear and autonomous [Battisti and Hirst, 1989], nonlinear, autonomous [Suarez and Schopf, 1988], or nonlinear, periodically forced equations [Tziperman et al., 1994; Münnich et al., 1990]. The latter will be our focus in this paper and can be formulated as a timedelay equation for the thermocline depth $h(t)$. The thermocline depth is a distinct layer of ocean in which temperature changes faster than it does in the layer above or below, thus separating the upper mixed layer from the deep ocean. One version of this equation has the form:

$$
\begin{aligned}
\frac{d h(t)}{d t}= & a \tanh \left(k h\left\{t-\frac{L}{2 c_{K}}\right\}\right) \\
& -d \tanh \left(k h\left\{t-\left[\frac{L}{c_{K}}+\frac{L}{2 c_{R}}\right]\right\}\right)+\cos (\Omega, t),
\end{aligned}
$$

where $k$ is an ocean-atmosphere coupling parameter, $c_{K}$ is the velocity of the wind-forced Kelvin mode, $L$ is the ocean basin width, and $c_{R}$ is the velocity of the Rossby wave. $\Omega$ is the frequency of the seasonal cycle, while $a, d$, and $c$ are constants. The cosine function in equation (1) accounts for the annual periodicity in the SST data. This delay differential equation has two time delays: $\tau_{1}=L / c_{K}+L / 2 c_{R}$, and $\tau_{2}=L / 2 c_{K}$. Here, $\tau_{1}$ is the summation over a time it takes a Rossby wave to travel from the middle of the ocean basin to the western boundary and then be reflected as a Kelvin wave, while $\tau_{2}$ is the transit time for the Kelvin wave which travels from the middle of the basin to reach the Eastern Pacific.

\section{Test for Determinism}

[6] The test for determinism (or more precisely, for lowdimensional deterministic dynamics) and for nonlinearity is based on reconstruction of the phase space from a scalar time series of length $N$ by time-delay embedding (see, for instance, Abarbanel [1996]) and is explained in depth in Kaplan and Glass [1993]. The method was recently successfully applied by us to explore the magnetospheric self-organization during magnetic storms and substorms [Živković and Rypdal, 2011, 2012]. When a system is low-dimensional deterministic, the direction of the trajectory (its tangent) is a function of the position in the reconstructed phase space. This means that trajectories emanating from points in a small neighborhood in phase space have almost parallel directions. On the other hand, corresponding trajectories in a stochastic or high-dimensional system have directions in a low-dimensional embedding space which are not uniquely dependent on the position in this space, and therefore, the tangent can have a different direction the next time it recurs to the same neighborhood. Let $b$ denote a small time increment and envisage a portion of phase space spanning the entire attractor divided into an enumerable set of small "boxes" of size corresponding to the length of the trajectory increment:

$$
\begin{gathered}
\Delta \mathbf{x}(b, t)=[x(t+b)-x(t), x(t+\tau+b)-x(t+\tau), \ldots, \\
x(t+(m-1) \tau+b)-x(t+(m-1) \tau)
\end{gathered}
$$

The tangent for the $k$ th pass of the trajectory through box $j$ is the unit vector $\mathbf{u}_{k, j}=\Delta \mathbf{x}_{k, j}(b, t) /\left|\Delta \mathbf{x}_{k, j}(b, t)\right|$. The estimated average displacement vector in the box is

$$
\mathbf{V}_{j}=\frac{1}{n_{j}} \sum_{k=1}^{n_{j}} \mathbf{u}_{k, j}
$$

where $n_{j}$ is the number of passes of the trajectory through box $j$. If the embedding dimension is sufficiently high and in the limit of vanishingly small box size (determined by the parameter $b$ ), the trajectory directions should be aligned and the length $V_{j} \equiv\left|\mathbf{V}_{j}\right|=1$. A condition for the validity of this assertion is that $\lambda b=1$, where $\lambda$ is the largest Lyapunov exponent. In this case $\mathbf{V}_{j}$ will not depend very much on the number of passes $n_{j}$, and $V_{j}$ will converge to 1 as $n_{j} \rightarrow \infty$. In contrast, for the trajectory of a random process, where the direction of the next step is completely independent of the past, $V_{j}$ will decrease with $n_{j}$ as $V_{j} \sim n_{j}^{-1 / 2}$. If the deterministic signal is contaminated by noise which dominates on short time scales, the test for determinism may yield a negative result if the box size is chosen too small. Hence, if this is suspected. it is recommended to vary the box size within the interesting range of time scales before drawing firm conclusions [Kaplan and Glass, 1993]. The degree of 
determinism of the dynamics can be assessed by exploring the dependence of $V_{j}$ on $n_{j}$. In practice, this can be done by computation of the average displacement vector length:

$$
L_{n} \equiv\langle V\rangle_{j n_{j}=n}
$$

where the average is done over all boxes with same number $n$ of trajectory passes.

[7] Let us recall that we are describing a test to distinguish signals described by low-dimensional dynamical systems, i.e., signals that in the continuous-time limit are solutions to differential equations and therefore continuous, and signals described as a stochastic process. The archetype of a random and continuous stochastic process is the Wiener process (Brownian motion), which in discrete time is a random walk. The random walk has random increments, and hence the displacement vectors $\Delta \mathbf{x}(t)$ in an $m$-dimensional embedding space will have random directions. Hence, for a random walk $L_{n \rightarrow \infty}=0$. For finite $n$, however, there will be a finite statistical spread of $V_{j}$, and as shown in Kaplan and Glass [1993], the average displacement of $n$ passes in $m$-dimensional phase space is

$$
L_{n}=R_{n} \equiv \frac{1}{\sqrt{n}}\left(\frac{2}{m}\right) 1 / 2 \frac{\Gamma[(m+1) / 2]}{\Gamma(m / 2)},
$$

where $\Gamma$ is the gamma function. The deviation in $\left\langle V_{j}\right\rangle$ between a given time series and a random walk can be characterized by a single number given by the weighted average over all boxes of the quantity,

$$
\Lambda(\tau) \equiv \frac{1}{\sum_{j} n_{j}} \sum_{j} n_{j} \frac{\left\langle V_{j}\right\rangle^{2}(\tau)-R_{n_{j}}^{2}}{1-R_{n_{j}}^{2}},
$$

where we have explicitly highlighted that the average displacement $\left\langle V_{j}\right\rangle(\tau)$ of the trajectory in the reconstructed phase space depends on the time delay $\tau$. For a completely deterministic signal we have $\Lambda(\tau)=1$, and for a completely

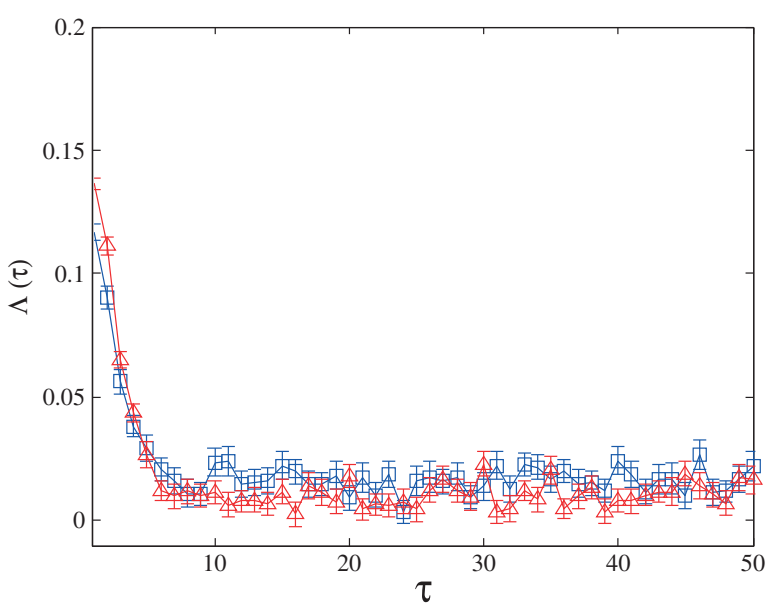

Figure 1. The $\Lambda(\tau)$ averaged over results computed from an ensemble of 10 realizations of the O-U process (squares) and for the realizations with randomized phases (triangles). Error bars denote standard errors of the mean $\Lambda(\tau)$ (which is $(1 / \sqrt{10}) \times$ the standard deviation of the distribution of the 10 samples). random signal $\Lambda(\tau)=0$; hence, this quantity can be considered as a measure of determinism.

[8] In Figure 1, we show $\Lambda(\tau)$ averaged over 10 numerical realizations of the Ornstein-Uhlenbeck $(\mathrm{O}-\mathrm{U})$ stochastic process, for embedding dimension $m=8$, and $b=1$. The $\mathrm{O}-\mathrm{U}$ process is described by the stochastic equation:

$$
d S_{t}=-\lambda S_{t}+\sigma d W_{t},
$$

where $W(t)$ is the Wiener process, $\lambda$ is a damping rate and $\sigma$ is a diffusion coefficient. It is a more physically realistic random process for many phenomena than the Wiener process, since the damping term - $\lambda S_{t}$ makes it bounded. In Figure 1, we also show mean $\Lambda(\tau)$ computed from the same 10 realizations, but after randomization of the phases of the Fourier coefficients. These realizations with randomized phases are called surrogate data. The phase randomization leaves the power spectral density, and hence the autocorrelation function, unchanged. Hence, $\Lambda(\tau)$ should also be unchanged for a random process, which Figure 1 demonstrates. On the other hand, for a signal from a low-dimensional chaotic system, which has to be nonlinear to be chaotic, the randomization of phases will destroy the nonlinear coupling between Fourier modes and make $\Lambda(\tau)$ more similar to a random signal, i.e., it will be reduced compared to $\Lambda(\tau)$ for the original signal. Examples of this were shown in Kaplan and Glass [1992, 1993]. Tests involving surrogate data can be thought of as a test of nonlinearity, but using $\Lambda(\tau)$ for this test will only reveal the nonlinearity if $\Lambda(\tau)$ for the original time series is large enough to reveal a low-dimensional, deterministic component. Hence, it can only distinguish nonlinear from linear dynamics when the dynamics is known to be deterministic and low-dimensional.

\section{Results}

[9] In this section, we analyze SOI and Niño 3 data. The Southern Oscillation index (SOI) analyzed here is computed using monthly mean sea level pressure anomalies at Tahiti and Darwin downloaded from the Climate and Global Dynamics Division of the National Center for Atmospheric Research. A description is found in Trenberth and Hoar [1996]. The Niño 3 data are the area-averaged SST from $5^{\circ} \mathrm{S}-5^{\circ} \mathrm{N}$ and $150^{\circ} \mathrm{W}-90^{\circ} \mathrm{W}$, recorded with monthly resolution between 1871 and 2008. It is retrieved from the Working Group of Surface Pressure of the Global Climate Observing System and has been derived from the sea ice and SST data set (HadISST1) [Rayner et al., 2003].

[10] We compute $\Lambda$ as a function of time-delay $\tau$, which is also used in the phase-space reconstruction procedure. The embedding dimension is $m=10$, and $b=1$, i.e., the box size is equal to the average distance between two successive points on the reconstructed phase-space trajectory. It has been shown in Kaplan and Glass [1993] that increasing embedding dimension can increase $\Lambda$ in deterministic systems, while it should not influence $\Lambda$ in stochastic systems. In principle, higher $m$ is better, but we also have to consider the number of data points available for the test, and this decreases with increasing $m$. In our analysis, $m=10$ seems a suitable choice of embedding dimension. Figure 2 shows $\Lambda(\tau)$ computed from the SOI (squares) and the ensemble mean of $\Lambda(\tau)$ computed from 50 surrogate time series obtained from the randomization of phases of the Fourier 


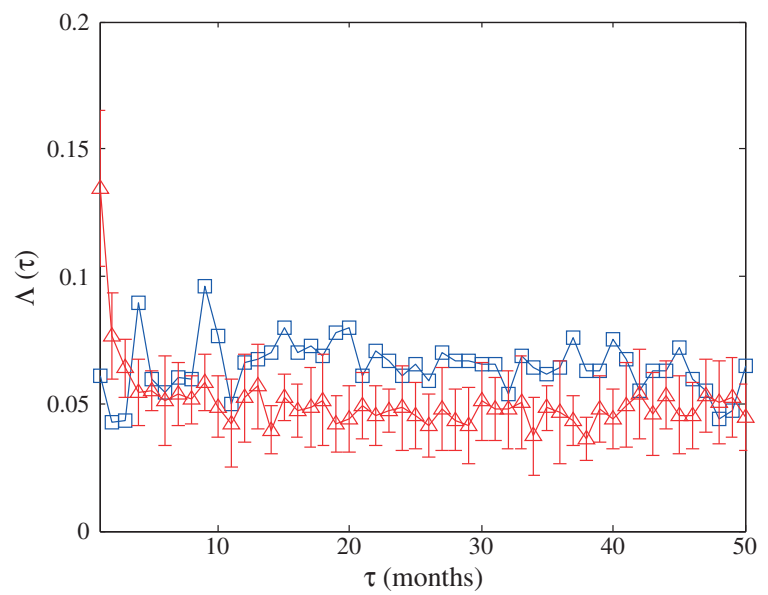

Figure 2. The $\Lambda(\tau)$ for the SOI time series (squares) and $\Lambda(\tau)$ averaged over 50 different phase-randomized versions of this time series (triangles). The error bars of the latter denote the standard deviation of $\Lambda(\tau)$ over the distribution of these 50 samples.

coefficients (triangles). The low values $(\Lambda<0.1)$ indicate a very weak deterministic component, although it is not as weak as for the O-U process shown in Figure 1. The reduction in $\Lambda$ when SOI is replaced by its surrogates is about $30 \%$ and is statistically significant. This somewhat ambiguous result may shed some light on the conflicting results reported in the literature, e.g., the opposite conclusion drawn by Binder and Wilches (2002) and Elsner and Tsonis [1993]. In principle, this could be because the SOI represents a nonlinear measurement function of the climate state, and hence not due to nonlinear dynamics, but this possibility was rejected by Elsner and Tsonis [1993]. We have confirmed their conclusion on this point by the application of a method similar to theirs, invoking our test of determinism rather than their nonlinear prediction algorithm. The idea is to start with a realization of a Gaussian white noise process and shuffle the data such that the amplitude at time $t$ has the same rank as the amplitude at time $t$ has in the SOI time series. This synthetic time series has the same overall appearance and correlation structure as the SOI record, but with a Gaussian amplitude distribution. We then compute the $\Lambda(\tau)$ for this time series and for an ensemble of surrogates obtained by the randomization of the phases of Fourier coefficients. The result for both the synthetic series and its surrogates are indistinguishable from those for the SOI and its surrogates. If the SOI series were the result of a nonlinear measurement function applied to an underlying Gaussian process, one would expect that the signature of nonlinearity would have been lost in the synthetic Gaussian record. This result is also consistent with the observation that the amplitude distribution of the original SOI record appears to be close to Gaussian, indicating the absence of distortion of the distribution due to a nonlinear measurement function.

[11] Although the SOI is a difference between two pressure anomalies, the way the anomalies are computed does not completely eliminate seasonal variation in the SOI. In Figure 3, the SOI climatology is plotted along with the Niño 3 climatology and with the sinusoidal climatology used in equation (1). Here, the climatology is defined as the mean overall data for every month of the year. One should expect

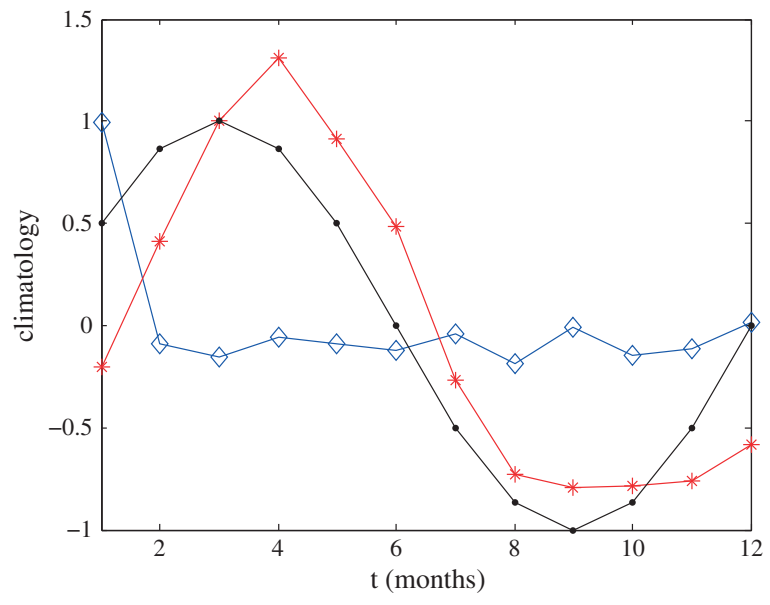

Figure 3. Climatology for Niño 3 (stars), SOI (diamonds), and the sinusoidal climatology used in equation (1) (dots).

the SOI climatology to be zero for all months of the year, but the figure reveals a deviation in January. This signifies that the SOI signal has a certain annual periodicity, which might be another reason why we observe some nonlinearity in the analysis of the SOI record in Figure 2. In Figure 4, we plot $\Lambda$ for the SOI when its climatology is subtracted together with the ensemble mean of $\Lambda$ computed for 50 corresponding surrogates. The test for determinism for this de-seasonalized SOI record gives results very similar to those for the O-U process in Figure 1, i.e., there is no sign of deterministic, low-dimensional, and nonlinear dynamics in the deseasonalized SOI data.

[12] The Niño 3 record does exhibit the anomalous January feature of the SOI, and the climatology is more similar to the sinusoidal seasonal forcing used in equation (1). The raw Niñõ 3 record is not an anomaly and contains the full seasonal cycle. This influences the estimate of $\Lambda(\tau)$ in two distinct ways. In Figure 5, we observe that $\Lambda(\tau)$ exhibits spiky dips at those $\tau$ where the autocorrelation function $r(\tau)$ has an extremum. This is a spurious feature of the

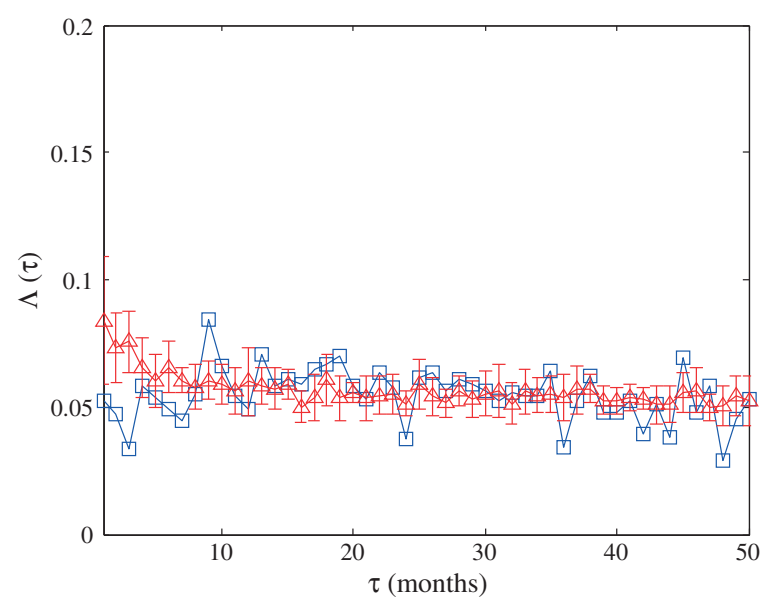

Figure 4. The $\Lambda(\tau)$ for the SOI time series with the climatology subtracted (squares) and $\Lambda(\tau)$ averaged over 50 different phase-randomized versions of this time series (triangles). The error bars of the latter denotes the standard deviation of $\Lambda(\tau)$ over the distribution of these 50 samples. 


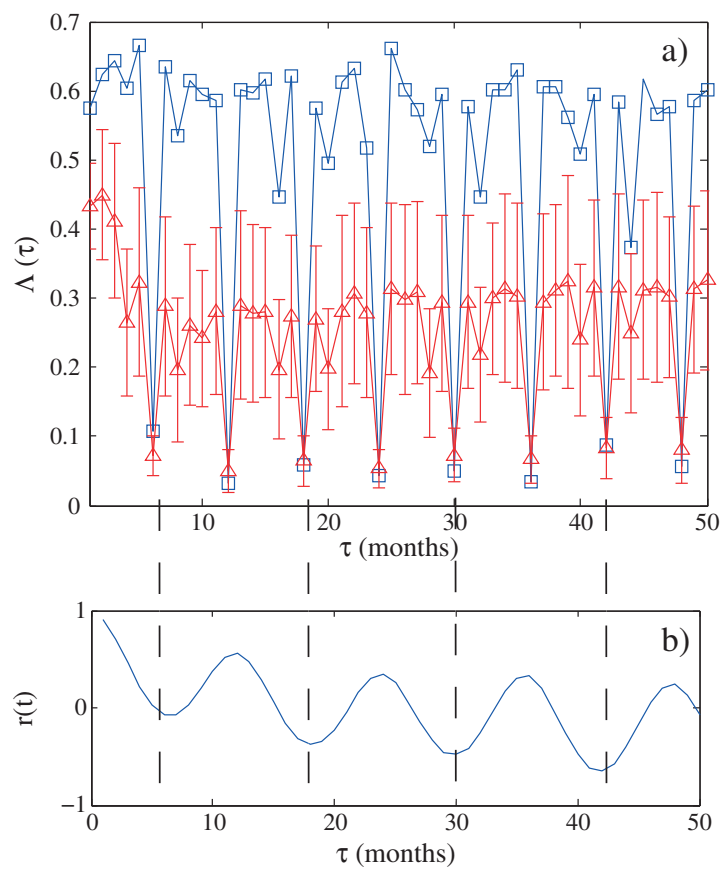

Figure 5. (a) The $\Lambda(\tau)$ for the Niño 3 time series (squares) and $\Lambda(\tau)$ averaged over 10 different phase-randomized versions of this time series (triangles). The error bars of the latter denotes the standard deviation of $\Lambda(\tau)$ over the distribution of these 10 samples. (b) autocorrelation function $r(\tau)$ for the Niño 3 time series.

technique when there is a cyclic component in the signal and is explained in Kaplan and Glass [1993]. The other distinct effect is that apart from the spurious dips, the determinism is high $(\Lambda>0.6)$, and nonlinearity is strong, since $\Lambda$ is reduced by more than $50 \%$ when the surrogate data are analyzed. Next, we subtract the climatology from the Niño 3 data and compute $\Lambda(\tau)$ again (Figure 6a). $\Lambda(\tau)$ is strongly reduced and the analysis of surrogates makes no discernible difference, confirming our findings from the de-seasonalized SOI time series. In the remainder of the paper, we will focus on the Niñõ 3 data since from a practical climatological viewpoint, the important aspects of ENSO are directly associated with the SST anomalies.

[13] Our results so far imply that the Niño 3 time series with the seasonal cycle subtracted can be described as a stochastic process similar to the O-U process for which results were presented in Figure 1. It does not imply, however, that the time-delay equation (1) has to be abandoned, since inclusion of a stochastic forcing term may give rise to such stochastic dynamics and produce results compatible with the test for determinism in Niño 3 data. In the following, we shall first show that a time-delay equation with stochastic forcing is able to produce time series with these properties. Next, we shall demonstrate that in the absence of stochastic forcing, the time-delay model tends to produce low-dimensional dynamics, even when seasonality is removed.

[14] In order to investigate the effect of stochastic forcing, we add a term $\sigma w(t)$, where $w(t)$ is a Gaussian white noise with unit variance, to equation (1) and solve the equation numerically with the parameters $a=0, d=1, k=100, c=1.3$, $\sigma=0.7$, and $\tau_{1}=0.2$. Figure $6 \mathrm{~b}$ shows $\Lambda(\tau)$ ensemble
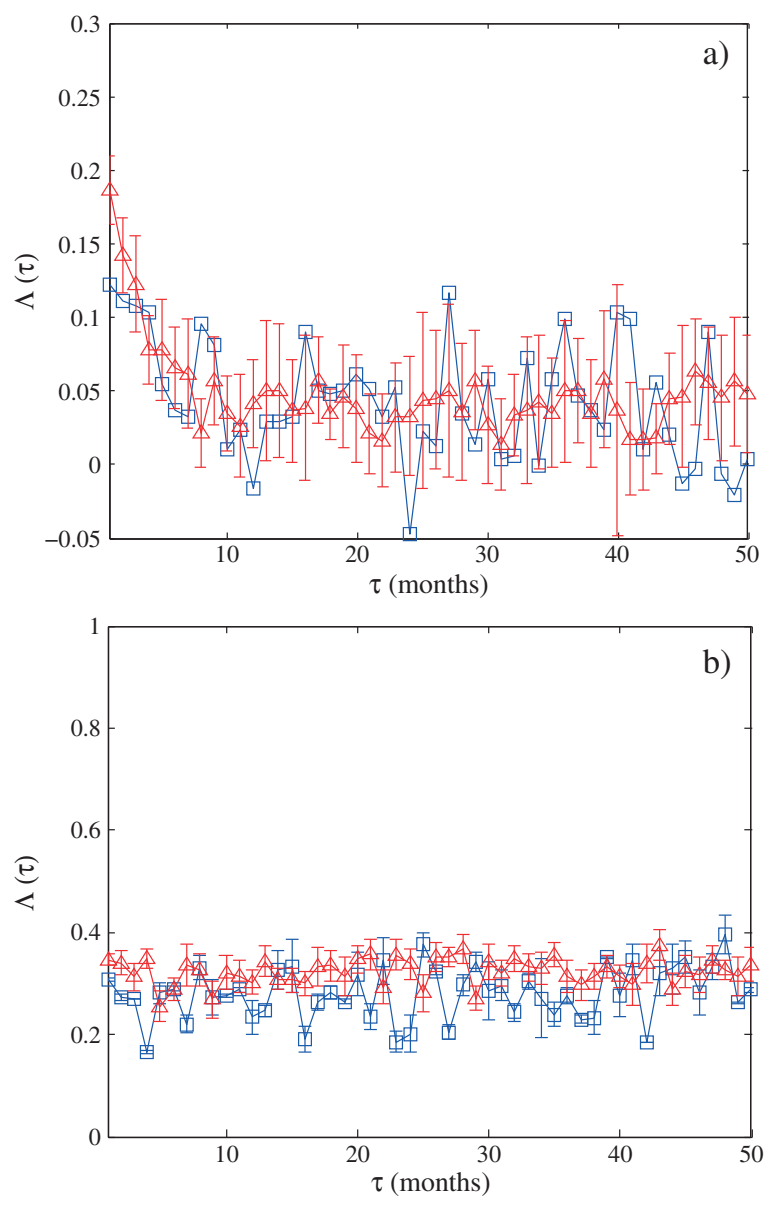

Figure 6. (a) The $\Lambda(\tau)$ for original Niño 3 time series with climatology subtracted (blue squares) and $\Lambda(\tau)$ averaged over 10 realizations of phase-randomized versions of this time series (red triangles). The error bars of the latter denotes the standard deviation of $\Lambda(\tau)$ over the distribution of these 10 samples. (b) $\Lambda(\tau)$ averaged over 10 realizations of time series generated by equation (1) with a white-noise stochastic forcing (squares), and the same for 10 phase-randomized versions (triangles). The climatology has been subtracted before computing $\Lambda(\tau)$. In (b), the error bars denote the standard deviation of the mean $\Lambda(\tau)$.

averaged over 10 realizations of the simulation after the climatology has been subtracted. In the same plot, we show $\Lambda(\tau)$ averaged over the 10 corresponding phase-randomized realizations. The low values of $\Lambda(\tau)$, and their nonresponsiveness to phase randomization, indicate that for these parameters the de-seasonalized solutions of the stochastically forced equation (1) have the character of a stochastic process similar to the de-seasonalized Niño 3 time series. A qualitative similarity is also apparent from Figure 7, where we plot a realization of this process along with the Niño 3 time series, both with the climatology subtracted. Both time series have been normalized to have unit variance. This similarity is also apparent when $\Lambda(\tau)$ is computed from the simulation without subtracting the seasonal cycle, as shown by comparing Figure 8 with Figure 5. The model described above, but without stochastic forcing $(\sigma=0)$, was studied by Ghil et al. [2008], who used the same choice of parameters $a, c$, and $d$ as above, but varied the parameters $k$ and $\tau_{1}$. They computed and plotted 

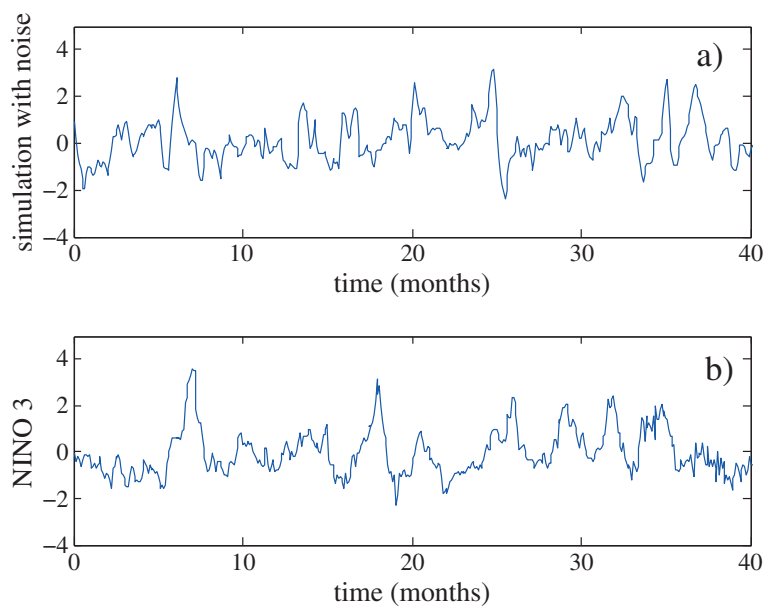

Figure 7. Time series analyzed in Figure 6. (a) Numerical realization of time-delay equation with stochastic forcing included and climatology subtracted. (b) Niño 3 index with climatology subtracted.

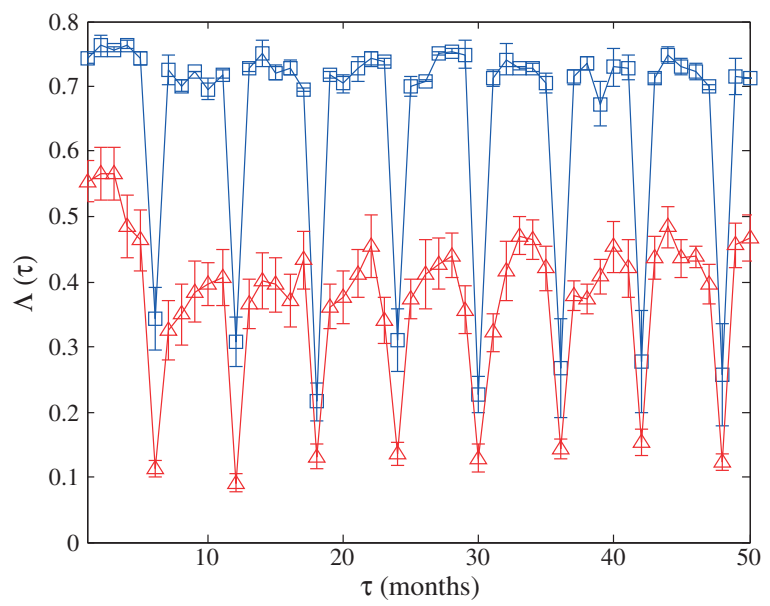

Figure 8. The $\Lambda(\tau)$ averaged over results computed from 10 simulated time series like that shown in Figure $7 \mathrm{a}$ with climatology included (blue squares) and after phase randomization (red triangles). The error bars denote the standard deviation of the mean $\Lambda(\tau)$.

the global maximum of the thermocline depth $h(t)$ as a function of $\tau_{1}$ and $k$, and observed discontinuities in this parameter space separating regions of regular solutions strictly governed by the seasonal forcing and more ENSO-like irregular solutions, indicating a structural instability of the model. In Figure 9a, we have plotted such a regular solution obtained for $\tau_{1}=0.4088$, and in Figure 9b, an irregular one on the other side of the parameter-space discontinuity: for $\tau_{1}=0.4198$. In both of these cases, $a=0, c=1, k=100$, and $d=1$. This irregular solution exhibits a seasonal cycle of variable strength interrupted by stronger episodical oscillations with some reminiscence of observed ENSO episodes. These episodes are phase-locked to the seasonal cycle, in agreement with the observations of Stein et al. [2011], but they appear at more regular intervals (3-4 years) and have shorter duration than real El Niño/La Niña events. Hence, the similarity to the Niño

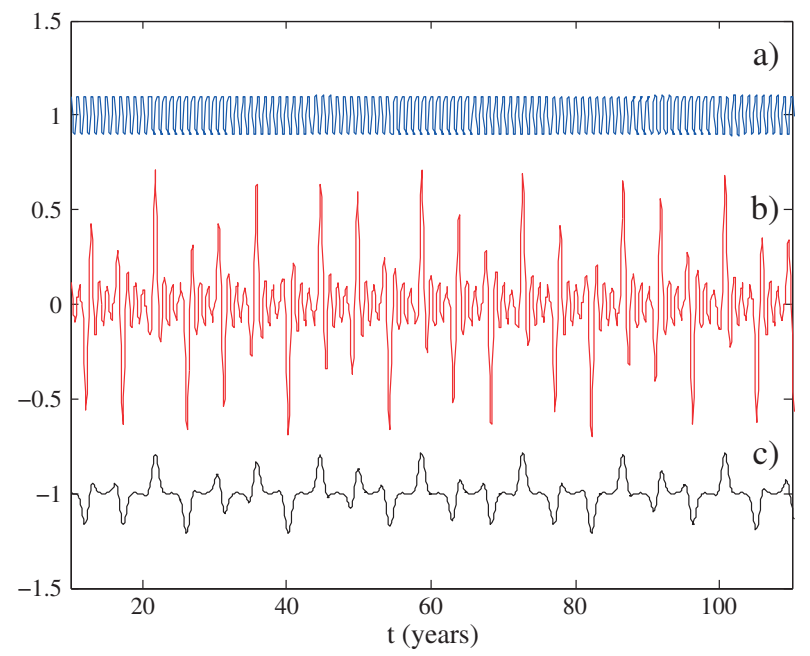

Figure 9. Simulation of time-delay equation with no stochastic forcing. (a) $\tau_{1}=0.4088$, (b) $\tau_{1}=0.4198$, (c) the signal in (b) de-seasonalized by wavelet filtering.
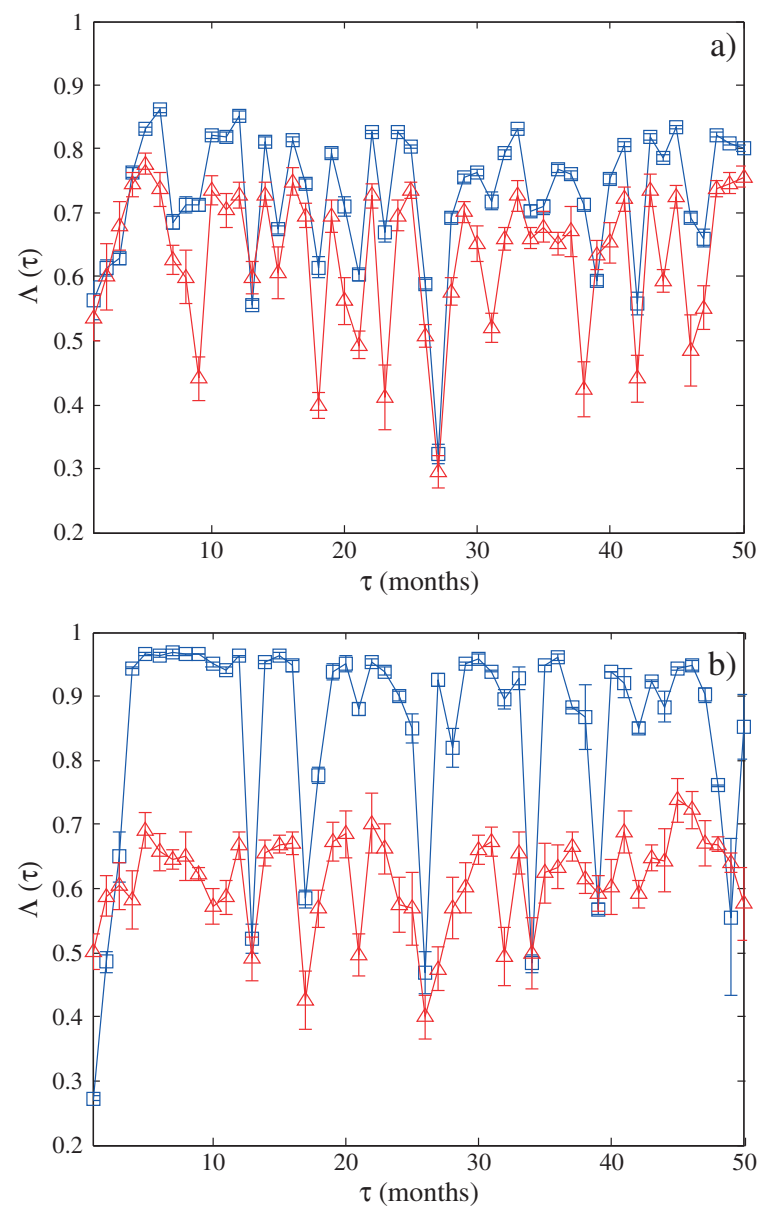

Figure 10. (a) The $\Lambda(\tau)$ averaged over results computed from 10 simulated time series like that shown in Figure $9 b$ (blue squares) and after phase randomization (red triangles). (b) $\Lambda(\tau)$ averaged over results computed from 10 simulated time series like that shown in Figure 9c (blue squares) and after phase randomization (red triangles). Error bars denote standard deviation of the ensemble mean. 
3 signal is not too convincing. However, as can be seen from Figure 7, the solution with stochastic forcing added exhibits more qualitative similarity to the Niño 3 signal than the signal in Figure 9b. This qualitative discrepancy between the model signal generated in the absence of stochastic forcing and the Niño 3 signal is also reflected by the test of determinism. Figure 10a shows $\Lambda(\tau)$ for the signal in Figure $9 \mathrm{~b}$ and for its surrogate time series. The determinism is higher than the one for the Niño 3 signal shown in Figure 5a, and it is less reduced by phase randomization. The former is obviously due to the higher degree of randomness apparent in the Niño 3 signal. The lack of reduction of determinism after phase randomization of the Figure $9 \mathrm{~b}$ signal is due to the strong seasonal oscillation in this signal. Since a major fraction of the power resides in the annual Fourier component, the phase randomization will change the phase of this component, but the signal will still have a deterministic appearance after this change. This is all quite trivial, so the crucial test is to compute determinism after de-seasonalization. For the signal in Figure 9b, subtraction of the climatology does not offer an effective filtering of the seasonal component, since its amplitude varies a lot. For the same reason, Fourier filtering is also ineffective. However, we can remove the seasonal component by wavelet filtering. In Figure 9c, we show the de-seasonalized signal
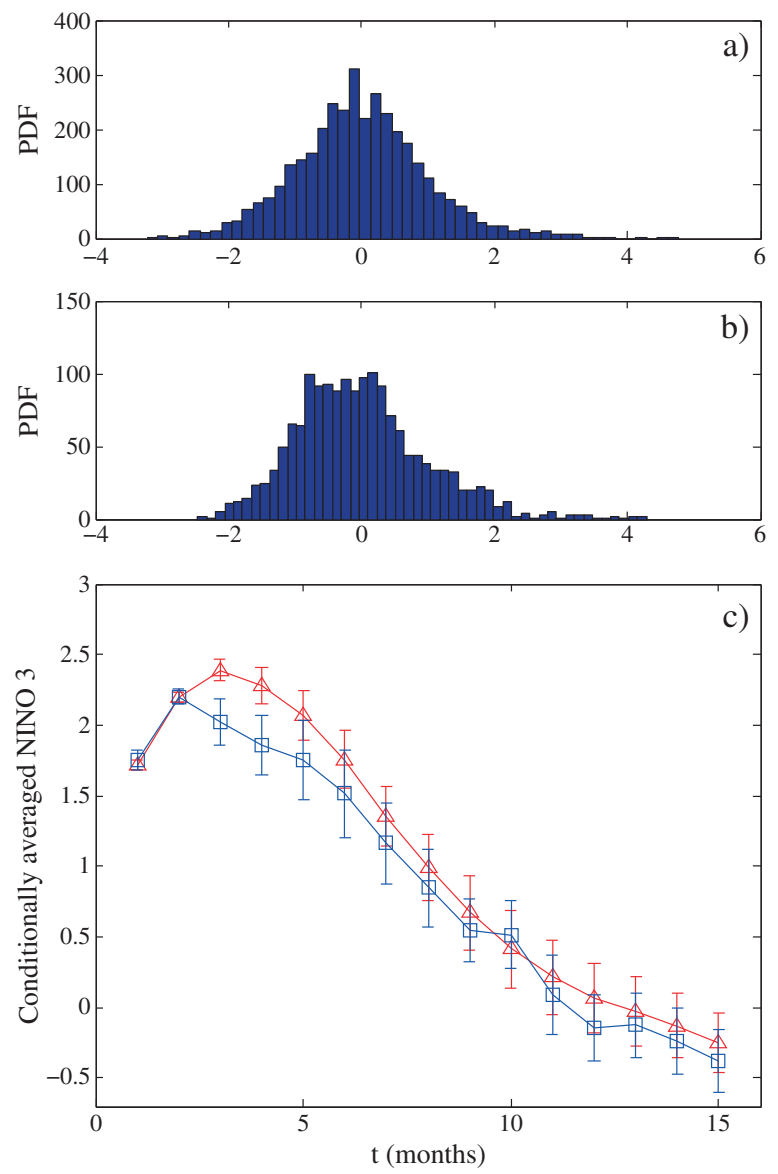

Figure 11. (a) Histogram of signal in Figure 7a. (b) Histogram of signal in Figure 7b. (c) Conditionally averaged evolution of ENSO signal after onset of ENSO episode. For simulation with stochastic forcing shown in Figure 7a (red triangles). For Niño 3 index shown in Figure 7b (blue squares). after the application of a Mexican-hat wavelet filter. This signal highlights the "true" ENSO episodes produced by the time-delay model without stochastic forcing. The computed determinism $\Lambda(\tau)$ of this signal is shown in Figure 10b. The determinism is very high and shows clearly that the ENSO episodes in this model are the result of deterministic, lowdimensional dynamics. The strong reduction of determinism after phase randomization demonstrates that this dynamics is low-dimensional and nonlinear. This is in strong contrast to the results shown in Figure 6 for de-seasonalized Niño 3 and signals generated by the time-delay model with stochastic forcing, which show signals dominated by a stochastic component.

[15] In the following, we go back to the time-delay simulation with stochastic forcing and demonstrate in two more examples its similarity to the Niño 3 data. In Figures 11a and $11 \mathrm{~b}$ we plot a histogram for the simulation and for the Niño 3 data after the climatology has been subtracted (the signals in Figure 7). The ENSO events contribute to the tails of these distributions, which are somewhat heavier on the positive side due to the relative strength of El Niño compared to La Niña. The average time evolution of ENSO events in Niño 3 and simulations can be investigated by means of a superposed-epoch analysis (conditional averaging). This analysis works as follows: Consider a signal $s(t)$. We define the onsets of ENSO events as the times $t_{n}, n=$ $1, \ldots, N$, for which the signal ascends through two standard deviations from the mean. Then we produce an ensemble of conditional signals $s_{n}(\delta t)=s\left(t_{n}+\delta t\right)$ and produce the conditional average $\left\langle s_{n}(\delta t)\right\rangle \equiv(1 / N) \sum_{n=1}^{N} s_{n}(\delta t)$. Figure 11c displays the average ENSO structure for Niño 3 (squares) and simulation (triangles) with climatology subtracted and shows that the simulation predicts fairly well the average evolution of ENSO up to at least 15 months after the onset of the episode.

\section{Conclusion}

[16] From a model for ENSO activity similar to the one studied here, Tziperman et al. [1995] came to the conclusion that depending on the strength of the coupling between the ocean and the atmosphere, the dynamics could undergo quasi-periodicity routes to chaos. The same authors suggested that the ENSO might be described as a low-dimensional chaotic dynamical system driven by the seasonal cycle, where the appearance of the chaos is due to the nonlinear resonance between the natural oscillator of the atmosphere-ocean coupling and the seasonal cycle. The irregular jumps of the state between different resonances was suggested to be the manifestation of chaos. Unfortunately, SST time series in the Eastern Pacific are too short for chaos to be proven by standard methods, and hence, it is a challenge to devise proper tests for these hypotheses. In recent years, there have been a large number of papers on dynamic, stochastic, and dynamic-stochastic modeling of the irregularity of ENSO (see e.g., the reviews of Dijkstra [2006] and Kleeman [2008]). The question of which types of description are more justified from the observational data, however, still remains controversial.

[17] In this paper we have applied a simple test for determinism to show that Niño 3 data, which is the proxy of SST in the Eastern Pacific, is most adequately described as a stochastic process after the seasonal cycle has been 
removed, implying that interannual ENSO variability is dominated by stochastic, not low-dimensional chaotic, dynamics. A similar conclusion was made by Binder and Wilches [2002], where a test for determinism was applied to the Southern Oscillation index (SOI) series. Despite the stochastic nature of the Niño 3 signal, we have demonstrated here that low-dimensional, deterministic dynamics may also be involved. The equatorial-wave equation from Tziperman et al. [1994] exhibits such dynamics without stochastic forcing, but by adding stochastic forcing to this equation and comparing determinism and average ENSO structure with those of Niño 3 data, we conclude that this statisticaldynamical model can reproduce important aspects of ENSO dynamics. This conclusion is also in the agreement with Franzke and Majda [2006], who demonstrated that the unresolved degrees of freedom in the system can be described by both deterministic and stochastic terms in low-order models. They conclude that the effective dynamics of the low-order system is driven by low-order chaotic as well as stochastic dynamics. Our work in this paper indicates that the deterministic dynamics is primarily associated with seasonal variability and, hence, that successful nonlinear prediction is possible only on time scales of a few months. Given the stochastic nature of the signals on interannual time scales, prediction of El Niño events several years ahead seems beyond reach.

\section{References}

Abarbanel, H. (1996), Analysis of Observed Chaotic Data, Institute for Nonlinear Science, Springer, New York.

Battisti, D. S., and A. C. Hirst (1989), Interannual variability in the tropical atmosphere-ocean system: Influence of the basic state and ocean geometry, J. Atmos. Sci., 46, 1687.

Bauer, S. T., and M. B. Brown (1992), Empirical low-order ENSO dynamics, Geophys. Res. Lett., 19, 2055-2058.

rBinder, P. M., and C. A. Wilches (2002), Absence of determinism in El-Niño Southern Oscillation, Phys. Rev. E, 65, doi:10.1103/PhysRevE.65.055207.

Dijkstra, H. A. (2006), The ENSO phenomenon: Theory and mechanisms, Adv. Geosci., 6, 3-15.
Dijkstra, H. A. (2010), Nonlinear Physical Oceanography. A Dynamical System Approach tothe Large Scale Ocean Circulation and El Niño, Kluwer.

Elsner, J. B., and A. A. Tsonis (1993), Nonlinear dynamics established in the ENSO, Geophys. Res. Lett., 20, 213-216.

Franzke, K., and A. J. Majda (2006), Low-order stochastic mode reduction for a prototype atmospheric GCM, J. Atmos. Sci., 63, 457-479, doi:10.1175/JAS3633.1.

Ghil, M., I. Zaliapin, and S. Thompson (2008), A delay differential model of ENSO variability: Parametric instability and the distribution of extremes, Nonlin. Processes Geophys., 15, 417.

Kaplan, D. T., and L. Glass (1992), Direct test for determinism in a time series, Phys. Rev. Lett., 68, 427.

Kaplan, D. T., and L. Glass (1993), Coarse-grained embedding of time series: Random walks Gaussian random processes, and deterministic chaos, Physica D, 64, 431.

Kleeman, R. (2008), Stochastic theories for the irregularity of ENSO, Phil. Trans. R. Soc., 366, 2511-2526.

Münnich, M., M. A. Cane, and S. E. Zebiak (1990), A study of self-excited oscillations of the tropical ocean-atmosphere system. Part 2: Nonlinear cases, J. Atmos. Sci., 48, 1238.

Rayner, N. A., D. E. Parker, E. B. Horton, C. K. Folland, L. V. Alexander, D. P. Rowell, E. C. Kent, and A. Kaplan (2003), Global analysis of sea surface temperature, sea ice, and night marine air temperature since the late nineteenth century, J. Geophys. Res., 108, 4407, doi:10.1029/ 2002JD002670.

Schreiber, T., and A. Schmitz (2000), Surrogate time series, Physica D, 142, 346-382.

Stein, K., A. Timmermann, and N. Schneider (2011), Phase synchronization of the El Niño-Southern oscillation with the annual cycle, Phys. Rev. Lett., 107, 128,501

Suarez, M. J., and P. S. Schopf (1988), A delayed action oscillator for ENSO, J. Atmos. Sci., 45, 3283.

Takens, F. (1981), Detecting strange attractors in fluid turbulence, in Dynamical Systems and Turbulence, edited by D. Rand and L. S. Young, Lect. Notes Math., 898, 366.

Trenberth, K. E., and T. J. Hoar (1996), The 1990-1995 El Nino-Southern Oscillation Event longest on record, Geophys. Res. Lett., 23, 57-60.

Tziperman, E., L. Stone, M. Cane, and H. Jarosh (1994), El Niño chaos: Overlapping of resonances between the seasonal cycle and the Pacific ocean-atmosphere oscillator, Science, 264, 72.

Tziperman, E., M. A. Cane, and S. E. Zebiak (1995), Irregularity and locking to the seasonal cycle in an ENSO prediction model as explained by the quasi-periodicity route to chaos, J. Atmos. Sci., 52, 293.

Živković, T., and K. Rypdal (2011), Low-dimensionality and predictability of solar wind and global magnetosphere during magnetic storms, J. Geophys. Res., 116, A10215, doi:10.1029/2011JA016547.

Živković, T., and K. Rypdal (2012), Organization of the magnetosphere during substorms, J. Geophys. Res., 117, A05212, doi:10.1029/ 2011JA016878 